\title{
On Smoluchowski Equations for Coagulation Processes with Multiple Absorbing States
}

\author{
Shay Gueron \\ Department of Mathematics \\ University of Haifa \\ Haifa, 31905, ISRAEL
}

\author{
Or Zuk \\ Department of Mathematics \\ Technion - Israel Institute of Technology \\ Haifa, 32000, ISRAEL
}

Version of: December 11, 2000

\begin{abstract}
Smoluchowski coagulation equations propose a model for the stochastic time evolution of a particles population in which particle clusters merge to form larger clusters, at some given rates. These equations represent the dynamics of the expected cluster size distribution. Since Smoluchowski equations were not derived as a rigorous description of the underlying stochastic process, their quality in this context is not obvious.

Here, we consider the case of a finite particles population and raise the following question: to what limit do the solutions of Smoluchowski equations converge as $t \rightarrow \infty$ ? In particular, we are concerned with the case where the population size is $N$ and the coagulation rates restrict the maximal group sizes to $D$. For $D=N$, the stochastic process has only one absorbing state, but if $D<N$ it may have many absorbing states. We demonstrate here that when the $D \approx N$, the solutions of Smoluchowski equations do not converge, as $t \rightarrow \infty$, to the expected cluster size distribution, but when $D \ll N$, the convergence is to a limit which is close to the exact solution.
\end{abstract}

\section{1 introduction}

A Coagulation process (CP) describes the stochastic time evolution of a population of particles distributed into groups (clusters) which coagulate at rates that depend only on the sizes of the interacting groups. Such processes and their applications have been studied extensively (see [3] for a thorough review). The process has been studied by means of the system of ODE's

$$
\dot{c}_{j}=\frac{1}{2} \sum_{k=1}^{j-1}\left(R_{j-k k} c_{j-k} c_{k}\right)-\sum_{k=1}^{\infty}\left(R_{j k} c_{j} c_{k}\right) \quad j=1,2, \ldots
$$

In (1), $c_{j}=c_{j}(t)$ denote the expected number of $j$-particle, and $R_{j k}$ represents the coagulation rate of clusters of size $j$ and $k$ into clusters of size $j+k$. These equations are named after Smoluchowski who formulated them in 1916 and 1917. They involve various mathematical problems which have been studied in many papers (see [3] for a review). When the coagulation rates are positive (i.e., $R_{j k}$ for $i, j>0$ ) and the particles population is assumed to be 
infinite, infinitely large clusters may appear at finite time (this phenomenon is called gelation). Thus, although the system (1) is formally a conservation law, the total mass is not always conserved. Further, under such conditions, the global existence, uniqueness and positiveness, of the solutions is not guaranteed.

The derivation of Smoluchowski equations is heuristic. Gueron [2] showed how equations similar to (1) can be viewed as a deterministic approximation for $c_{j}(t)$ if one ignores the effects of correlations. The consequences of neglecting these correlations may be significant, and therefore the value of Smoluchowski equations as an approximation for the expectation of the stochastic process is not clear. To emphasize the difficulty of ignoring correlations, we mention the case of coagulation-fragmentation processes where the model integral equation (the continuous analog of Smoluchowski equations) was shown to deviate from the exact solution [1].

In this paper we are concerned with the relation between the stochastic CP's and Smoluchowski equations for a finite particles population. For positive coagulation rates, which are those studied in the literature, the stochastic process has only one absorbing state: it always terminates with a single cluster containing the whole population. However, when the coagulation rates bound the largest cluster that can be generated, the CP may have many absorbing states. In this context, our questions are the following: to what limit do the solutions of Smoluchowski equations converge as $t \rightarrow \infty$ ? Does this limit approximate the expected steady state cluster size distribution? How are the multiple absorbing states being accounted for?

\section{The Coagulation Process}

\subsection{Preliminaries and definitions}

Consider a population of $N$ particles found at time $t=0$, at the "solitary state" where each particle forms a group of size 1 . The population undergoes stochastic evolution of coagulation events where any two groups can merge into a larger one. This CP is a time-homogeneous interacting particle system whose state space is (included in) the set $\Omega=\Omega_{N}=\{\eta\}$ of all partitions of $N$. We denote a partition of $N$ into $n_{i}$ summands of size $i, i=1,2, \ldots, N$, by $\eta=\left(n_{1}, n_{2}, \ldots, n_{N}\right)$, where $n_{i} \geq 0$ and $\sum_{i=1}^{N} i n_{i}=N$. In these notations, the initial state at $t=0$ is $\eta_{0}=(N, 0,0, \ldots, 0)$. Assuming mass action kinetics, we model the total merging intensity, $\Psi(i, j ; \eta)$, at the state $\eta=\left(n_{1}, n_{2}, \ldots, n_{N}\right) \in \Omega_{N}$ by 


$$
\begin{array}{r}
\Psi(i, j ; \eta)=\Psi\left(i, j ; n_{i}, n_{j}\right)=\psi(i, j)\left(n_{i} n_{j}\right), \quad i \neq j, \quad 2 \leq i+j \leq N \\
\Psi(i, i ; \eta)=\Psi\left(i, i ; n_{i}, n_{i}\right)=\psi(i, i) \frac{n_{i}\left(n_{i}-1\right)}{2}, \quad 2 \leq 2 i \leq N
\end{array}
$$

In (2), the rates of the infinitesimal (in time) transitions depend only on the sizes of the interacting groups. For $i$ and $j$ such that $1 \leq i, j \leq N-1$ and $2 \leq i+j \leq N$, the merging rate of two groups of sizes $i$ and $j$ into one group of size $i+j$ is denoted by $\psi(i, j)$, where $\psi(i, j)$ is some function satisfying $\psi(i, j)=\psi(j, i) \geq 0$. The CP is a non-ergodic Markov chain on $\Omega_{N}$ : from the initial state $\eta_{0}$, it eventually reaches an absorbing state.

We denote the set of all absorbing states of the process by $A$. For each state $\eta$, we denote the probability of being in $\eta$ at time $t$ by $\pi_{\eta}(t)$, and the probability of being in $\eta$ when $t \rightarrow \infty$ by $\pi_{\eta}$. The CP is non-ergodic Markov chain, and therefore the existence of such a limit is guaranteed. Clearly $\pi_{\eta}>0$ if and only if $\eta \in A$.

Our study concerns the resulting expected groups size distribution $f=f(i ; N)$. Here, $f(i ; N)$ denotes the expected number of groups of size $i$, when $t \rightarrow \infty$. The expected groups size distribution is given by

$$
f(i ; N)=\sum_{\eta \in \Omega_{N}} \pi_{\eta} n_{i}(\eta)=\sum_{\eta \in A} \pi_{\eta} n_{i}(\eta) \quad i=1,2, \ldots, N
$$

The time dependent groups size distribution is defined analogously. Note that mass conservation implies

$$
\sum_{i=1}^{N} i f(i, N)=N
$$

To identify the states in $\Omega_{N}$ we sort them lexicographically and use $\# \eta$ to denote the ordinal number of the state $\eta$.

\subsection{Coagulation processes with multiple absorbing states}

Suppose the coagulation kernel is strictly positive, that is, $\psi(i, j)>0$ for $i, j>0$ such that $i+j \leq N$. Then, independently of the initial condition, the process terminates only when all the particles are found in one cluster of size $N$. In other words, $\eta=(0,0, \ldots, 0,1)$ is the only absorbing state. 


\section{D-truncated CP's}

We define here another class of CP's, which we call D-truncated CP's, where $\psi(i, j)>0 \Longleftrightarrow$ $i+j \leq D$ for some $D \leq N$. In D-truncated CP's (starting from $\eta_{0}$ ) the size of a group never exceeds $D$. Accordingly, the process may have many absorbing states as shown the following examples.

\subsection{Direct computation of the group size distribution}

The group size distribution $f(i ; N)$ is a functional of the probability distribution $\pi$. One way to compute $f(i ; N)$ is to compute $\pi$ first, and to obtain $f(i ; N)$ from (3).

To compute the probability distribution $\pi$ we construct the transition rate matrix $B$, whose entries are determined as follows. A transition accounting for the coagulation of groups of size $i \neq j$ moves the population from a state $\eta=\left(n_{1}, n_{2}, \ldots, n_{N}\right)$ to the state $\xi=$ $\left(n_{1}, n_{2}, \ldots, n_{i}-1, \ldots, n_{j}-1, \ldots, n_{i+j}+1, \ldots, n_{N}\right)$. In this case, $B(\# \eta, \# \xi)=\psi(i, j) n_{i}(\eta) n_{j}(\eta)$. A transition representing the coagulation of two groups of size $i$ moves the population from a state $\eta=\left(n_{1}, n_{2}, \ldots, n_{N}\right)$ to the state $\xi=\left(n_{1}, n_{2}, \ldots, n_{i}-2, \ldots, n_{2 i}+1, \ldots, n_{N}\right)$. In this case, $B(\# \eta, \# \xi)=\frac{1}{2} \psi(i, i) n_{i}(\eta)\left(n_{i}(\eta)-1\right)$. For all other pairs $\eta, \xi$ such that $\eta \neq \xi$, the transition from $\eta$ to $\xi$ cannot occur, and therefore $B(\# \eta, \# \xi)=0$. Since the rate of leaving the state $\eta$ is the sum of all the rates of transitions emanating from $\eta$, the diagonal entries of $B$ are determined by

$$
B(\# \eta, \# \eta)=-\sum_{\eta \neq \xi \in \Omega_{N}} B(\# \eta, \# \xi)
$$

With these definitions, the probability distribution $\pi(t)$ is

$$
\pi(t)=\eta_{0} \times e^{B t}
$$

To compute $\pi$ (i.e., the limit as $t \rightarrow \infty$ ) we generate a new matrix, $C$, in a way that for any $\eta \neq \xi, C(\# \eta, \# \xi)$ is the transition probability from $\eta$ to $\xi$. This is done in the following way:

1. If $B(\# \eta, \# \eta) \neq 0$, then row number $\# \eta$ in $C$ is obtained by dividing row number $\# \eta$ in $B$ by $-B(\# \eta, \# \eta)$.

2. If $B(\# \eta, \# \eta)=0, \eta$ is an absorbing state, and the entire row of $B$ is 0 . In this case we set $C(\# \eta, \# \eta)=-1$ and the other entries of this row remain 0 .

For each absorbing state $\eta \in A$ we define $1_{\eta}$ as a vector of length $\left|\Omega_{N}\right|$, whose entries are 0 except for entry number $\# \eta$ which is set to be -1 . We now consider the linear system

$$
S(\eta): \quad C x=1_{\eta}
$$


and note that $\pi_{\eta}$ is the value of $x_{\# \eta_{0}}$ in the solution of $S(\eta)$. Thus, in order to find $\pi$, we have to solve $C x=1_{\eta}$, for every $\eta \in A$ (this requires one Gauss elimination with multiple free columns). The solution when the initial condition is not $\eta_{0}$ can be obtained from this system as well.

\subsection{The Smoluchowski equations}

Smoluchowski equations are an attempt to approximate the group size distribution by forming a self contained system of ODE's with the unknowns $f_{i}=f_{i}(t)$, where $f_{i}$ "represents" $f(i ; N)$. Following [2], the exact evolution equations for $f_{i}(t)$ read

$$
\begin{gathered}
\frac{d f_{i}}{d t}=\sum_{k=1, k \neq \frac{i}{2}}^{i-1} f_{k} f_{i-k} \psi(k, i-k)+\frac{1}{2} f_{\frac{i}{2}}\left(f_{\frac{i}{2}}-1\right) \psi\left(\frac{i}{2}, \frac{i}{2}\right)_{(i e v e n)}- \\
\sum_{k=1, k \neq i}^{D-1} f_{i} f_{k} \psi(i, k)-f_{i}\left(f_{i}-1\right) \psi(i, i)_{(2 i \leq D)}+\operatorname{CORR}_{i}, \quad(i=1,2, \ldots, D)
\end{gathered}
$$

with initial conditions $f_{1}=N$ and $f_{i}=0$ otherwise. Here, $C O R R_{i}$ are the correlation terms, and so far, $f_{i}$ and $f(i ; N)$ are the same (see [2]). To obtain a self contained system of equations (Smoluchowski type equations) for the unknowns $f_{i}$, we ignore the $C O R R_{i}$ terms that depend explicitly on the distribution $\pi_{i}$.

For computing the equilibrium, we take $\frac{d f_{i}}{d t}=0, i=1,2, \ldots, N$, in (8) and obtain a system of quadratic equations. Any steady state of the process is a solution of this system.

The question we address here concerns the comparison between limit, when $t \rightarrow \infty$, of $f_{i}$, the solution of the Smoluchowski system, and $f(i ; N)$, the expected groups size distribution (which we can compute directly for small populations).

\section{Example 1: $\mathrm{CP}$ with a small population and one absorbing state}

In our first example we take $N=5$, and $\psi(i, j) \equiv 1$. The state space $\Omega_{5}$ has 7 states,listed in lexicographic order

$\Omega_{5}=\{(0,0,0,0,1),(0,1,1,0,0),(1,0,0,1,0),(1,2,0,0,0),(2,0,1,0,0),(3,1,0,0,0),(5,0,0,0,0)\}$

There is only one absorbing state, namely $\eta=(0,0,0,0,1)$. The transition rates matrix $B$ is 


$$
B=\left(\begin{array}{rrrrrrc}
0 & 0 & 0 & 0 & 0 & 0 & 0 \\
1 & -1 & 0 & 0 & 0 & 0 & 0 \\
1 & 0 & -1 & 0 & 0 & 0 & 0 \\
0 & 2 & 1 & -3 & 0 & 0 & 0 \\
0 & 1 & 2 & 0 & -3 & 0 & 0 \\
0 & 0 & 0 & 3 & 3 & -6 & 0 \\
0 & 0 & 0 & 0 & 0 & 10 & -10
\end{array}\right)
$$

and the "normalized" matrix $C$ is

$$
C=\left(\begin{array}{rrrrrrr}
-1 & 0 & 0 & 0 & 0 & 0 & 0 \\
1 & -1 & 0 & 0 & 0 & 0 & 0 \\
1 & 0 & -1 & 0 & 0 & 0 & 0 \\
0 & \frac{2}{3} & \frac{1}{3} & -1 & 0 & 0 & 0 \\
0 & \frac{1}{3} & \frac{2}{3} & 0 & -1 & 0 & 0 \\
0 & 0 & 0 & \frac{1}{2} & \frac{1}{2} & -1 & 0 \\
0 & 0 & 0 & 0 & 0 & 1 & -1
\end{array}\right)
$$

For finite time, the group size distribution is calculated by using (12) and (6). The steady state group size distribution (i.e., at $t \rightarrow \infty)$, computed by $(7)$, is $f_{\text {exact }}=(0,0,0,0,1$ ) (it corresponds with having only one absorbing state).

The Smoluchowski equations for this case read

$$
\begin{gathered}
\dot{f}_{1}(t)=-f_{1}(t)\left(f_{1}(t)-1\right)-f_{1}(t) f_{2}(t)-f_{1}(t) f_{3}(t)-f_{1}(t) f_{4}(t) \\
\dot{f}_{2}(t)=-f_{1}(t) f_{2}(t)-f_{2}(t)\left(f_{2}(t)-1\right)+\frac{1}{2} f_{1}(t)\left(f_{1}(t)-1\right)-f_{2}(t) f_{3}(t) \\
\dot{f}_{3}(t)=-f_{1}(t) f_{3}(t)+f_{1}(t) f_{2}(t)-f_{2}(t) f_{3}(t) \\
\dot{f}_{4}(t)=\frac{1}{2} f_{2}(t)\left(f_{2}(t)-1\right)+f_{3}(t) f_{1}(t)-f_{1}(t) f_{4}(t) \\
\dot{f}_{5}(t)=f_{1}(t) f_{4}(t)+f_{2}(t) f_{3}(t)
\end{gathered}
$$

with the initial conditions $f_{1}(0)=5, f_{2}(0)=f_{3}(0)=f_{4}(0)=f_{5}(0)=0$.

The equations of (11) are, by definition, functionally dependent: adding up $i$ times equation number $i$, for $i=1,2, \ldots, 5$, gives an identity $\dot{f}_{1}(t)+2 \dot{f}_{2}(t)+3 \dot{f}_{3}(t)+4 \dot{f}_{4}(t)+5 \dot{f}_{5}(t)=0$, which reflects the fact that the system (11) is a conservation law.

To compare the solution of Smoluchowski system (11) with the exact expected group size distribution, we solved (11) numerically. Fig. 1 shows the trajectory of $f_{2}(t)$ (panel a), and $f_{4}(t)$ (panel b) for $0 \leq t \leq 30$, compared with the exact solution (which was obtained from (6) and the definition of the expectation). As one can see, the wiggling solution of (11) is not 
a good approximation to the exact solution. Further, note that the numerical trajectory of (11) dips below 0 , which renders it completely irrelevant.

Propagating the numerical trajectory of (11) to large $t$, enables us to approximate numerically the limit to which the system converges, and the result is $f_{\text {Smoluchowski }}=(1,0,0,0,0.8)$. Recalling that the exact expected equilibrium group size distribution is $f_{\text {exact }}=(0,0,0,0,1)$, we conclude that $f_{\text {Smoluchowski }}$ is not a good approximation.

\section{Example 2: D-truncated $\mathbf{C P}(D \approx N) \mathbf{C P}$ with a small population and multiple absorbing states}

We now study a D-truncated CP. We take $N=5, D=4, \psi(2,3)=\psi(3,2)=\psi(1,4)=$ $\psi(4,1)=0$, and $\psi(i, j)=1$ for all other $i, j$. The state space is $\Omega_{5}$, which was listed above. Unlike the case with Example 1, we have here two absorbing states. These are

$$
A=\{\eta=(0,1,1,0,0), \xi=(1,0,0,1,0)\}
$$

The transition rates matrix $B$ is

$$
B=\left(\begin{array}{rrrrrrr}
0 & 0 & 0 & 0 & 0 & 0 & 0 \\
0 & 0 & 0 & 0 & 0 & 0 & 0 \\
0 & 0 & 0 & 0 & 0 & 0 & 0 \\
0 & 2 & 1 & -3 & 0 & 0 & 0 \\
0 & 1 & 2 & 0 & -3 & 0 & 0 \\
0 & 0 & 0 & 3 & 3 & -6 & 0 \\
0 & 0 & 0 & 0 & 0 & 10-10
\end{array}\right)
$$

and the normalized matrix $C$ is

$$
C=\left(\begin{array}{rrrrrrr}
-1 & 0 & 0 & 0 & 0 & 0 & 0 \\
0 & -1 & 0 & 0 & 0 & 0 & 0 \\
0 & 0 & -1 & 0 & 0 & 0 & 0 \\
0 & \frac{2}{3} & \frac{1}{3} & -1 & 0 & 0 & 0 \\
0 & \frac{1}{3} & \frac{2}{3} & 0 & -1 & 0 & 0 \\
0 & 0 & 0 & \frac{1}{2} & \frac{1}{2} & -1 & 0 \\
0 & 0 & 0 & 0 & 0 & 1 & -1
\end{array}\right)
$$

To compute the probability of being absorbed in $\eta=(0,1,1,0,0)$ (the first absorbing state of $A)$, we set $1_{\eta}=(0,-1,0,0,0,0,0)$ and solve the system $S(\eta): C x=1_{\eta}$. The solution is

$$
x=\left(0,1,0, \frac{2}{3}, \frac{1}{3}, \frac{1}{2}, \frac{1}{2}\right)
$$


From (14) it follows that $\pi_{\eta}=x_{7}=\frac{1}{2}$. Recalling that there are only two absorbing states, it follows that $\pi_{\xi}=1-\pi_{\eta}=\frac{1}{2}$, i.e., the probability of being absorbed in either one of the absorbing states equals $\frac{1}{2}$. The resulting steady state group size distribution, computed by $(3)$, is $f_{\text {exact }}=(0.5,0.5,0.5,0.5,0)$.

The Smoluchowski equations in this case read

$$
\begin{gathered}
\dot{f}_{1}(t)=-f_{1}(t)\left(f_{1}(t)-1\right)-f_{1}(t) f_{2}(t)-f_{1}(t) f_{3}(t) \\
\dot{f}_{2}(t)=-f_{1}(t) f_{2}(t)-f_{2}(t)\left(f_{2}(t)-1\right)+\frac{1}{2} f_{1}(t)\left(f_{1}(t)-1\right) \\
\dot{f}_{3}(t)=-f_{1}(t) f_{3}(t)+f_{1}(t) f_{2}(t) \\
\dot{f}_{4}(t)=\frac{1}{2} f_{2}(t)\left(f_{2}(t)-1\right)+f_{3}(t) f_{1}(t) \\
\dot{f}_{5}(t)=0
\end{gathered}
$$

with the initial conditions $f_{1}(0)=5, f_{2}(0)=f_{3}(0)=f_{4}(0)=f_{5}(0)=0$.

To compare the solution of (15) with the exact expected group size distribution, the system (15) was solved numerically. Fig. 2 shows the numerical trajectory of $f_{2}(t)$ (panel a), and $f_{4}(t)$ (panel b) for $0 \leq t \leq 30$, compared with the exact solution. As with the previous example, the wiggling solution of (15) is not a good approximation to the exact solution, and the numerical trajectory of (15) dips below 0 .

By propagating the numerical solution of (15) to large $t$, we find (numerically) that the solution of the Smoluchowski system converges to $f_{\text {Smoluchowski }}=(1,0,0,1,0)$. It fails to approximate the exact solution $f_{\text {exact }}=(0.5,0.5,0.5,0.5,0)$. Moreover, it is easy to check (by substitution) that the exact solution $f_{\text {exact }}=(0.5,0.5,0.5,0.5,0)$ does not even satisfy the Smoluchowski equilibrium equations.

Note that in the given example, the numerical solution of Smoluchowski system converges to one of the two absorbing states $(1,0,0,1,0)$, whereas the expected equilibrium group size distribution is a nontrivial linear combination of them. However, in general, the Smoluchowski system does not necessarily converge to an absorbing state. For example, with the initial condition $f_{\text {Smoluchowski }}=(3,1,0,0,0)$, the solution of $(15)$ converges to $\approx(0,1,0.5787,0.3159,0)$. This solution is not an absorbing state and not even a linear combination of the two absorbing states. 


\section{Example 3: D-truncated CP $(D \ll N)$ with a large population and multiple absorbing states and}

Here we study a D-truncated CP with a large population and $D \ll N$. We take $N=100$, $D=3, \psi(1,1)=\psi(1,2)=\psi(2,1)=1$ with $\psi(i, j)=0$ otherwise. Here, we cannot use $\Omega_{100}$ directly because it too large. However, the relevant state space (containing only states with group of size not exceeding 3 ) has only 884 states (we do not list these states here). Also, there are 18 absorbing states, listed in lexicographic order:

$A=\{(0,2,32),(0,5,30),(0,8,28),(0,11,26),(0,14,24),(0,17,22),(0,20,20),(0,23,18),(0,26,16)$,

$(0,29,14),(0,32,12),(0,35,10),(0,38,8),(0,41,6),(0,44,4),(0,47,2),(0,50,0),(1,0,33)\}$

To compute $\pi$, we solve the $884 \times 884$ linear system $(7)$ with 18 free columns (one for each absorbing state). The resulting group size distribution is $f_{\text {exatc }}=(0.0091,7.9031,28.0616)$.

The Smoluchowsky equations this case reads

$$
\begin{gathered}
\dot{f}_{1}(t)=-f_{1}(t)\left(f_{1}(t)-1\right)-f_{1}(t) f_{2}(t) \\
\dot{f}_{2}(t)=-f_{1}(t) f_{2}(t)+\frac{1}{2} f_{1}(t)\left(f_{1}(t)-1\right) \\
\dot{f}_{3}(t)=f_{1}(t) f_{2}(t)
\end{gathered}
$$

with the initial conditions $f_{1}(0)=100, f_{2}(0)=f_{3}(0)=0$. This system was solved numerically, and the solution was propagated to large $t$ in order to approximate the equilibrium group size distribution to which the Smoluchowsky system converges as $t \rightarrow \infty$. The resulting limit is $f_{\text {Smoluchowski }}=(0.0000,6.4703,29.0198)$. This distribution is a fairly reasonable approximation to the exact solution (note also that if we substitute the exact solution into the Smoluchowsky equilibrium system, we get relatively small deviations from zero.)

\section{Larger populations}

Our conjecture is that as the population size $N$ grows, the Smoluchowsky equations (at least at equilibrium) provide a better approximation to the exact expected group size distribution. Verifying this conjecture is difficult because computing the exact solution directly is unfeasible for large values of $N$, due to the large state space and the corresponding dimensions of the transition rates matrix. To replace the unfeasible direct computation, we used a Monte Carlo method to simulate the actual stochastic process (see [2] for details). Simulation of the Dtruncated CP with $N=900, D=3, \psi(1,1)=\psi(1,2)=\psi(2,1)=1$ with $\psi(i, j)=0$ 
otherwise, gave $f_{\approx \text { exact }}=(0.0000,69.3329,253.7781)$. The Smoluchowsky system with these parameters yields $f_{s m}=(0.0000,67.9305,254.7130)$, which is a good approximation of the exact solution.

\section{Concluding remarks}

Smoluchowsky equations are not a good model for D-truncated CP's when $D \approx N$ (or $D=N)$. This is due to the correlation between relatively large interacting groups (see [2] for details). For small populations, we always have $D \approx N$ (or $D=N$ ), and we demonstrated that Smoluchowski equations produce a poor approximation to the stochastic CP. With nonpositive coagulation kernels, there are several absorbing states. The Smoluchowski system does not necessarily converge to the exact solution, neither to one of the absorbing states, or to a linear combination of absorbing states, and the solutions are not positive. The same occurs for strictly positive coagulation kernels.

For a large population and $D \ll N$, we gave an example where the Smoluchowski system converges, as $t \rightarrow \infty$, to a good approximation of the correct solution. We conjecture that this is the case for finite populations with $D \ll N$.

The classical study of CP's deals with strictly positive coagulation kernels and $N=\infty$, and is modeled by the infinite system of OD's (1). Many of the mathematical difficulties and phenomena associated with this model (e.g., violation of mass conservation) do not correspond to analogous phenomena in a finite particles population. Note that the classical study allows for only one absorbing state: one cluster containing the whole population. Therefore, in our terminology, the classical Smoluchowski equations can be viewed as the limit as $N \rightarrow \infty$ of a D-truncated CP where $D=N$. Recalling that for finite populations, the Smoluchowski equations for D-truncated CP's with $D=N$ produce a poor approximation to the exact group size distribution, illustrates an intrinsic difficulty.

\section{References}

[1] R. Durrett, B. L. Granovsky, S. Gueron. Equilibrium behavior of the reversible coagulation-fragmentation processes. Journal of Theoretical Probability. 12:447-474 (1999).

[2] S. Gueron. The Steady-state distributions of coagulation-fragmentation processes. Journal of Mathematical Biology 37:1-27 (1998). 
[3] D. Aldous. Deterministic and stochastic models for coalescence (aggregation, coagulation): a review of the mean-field theory for probabilists. Bernoulli 5:3-48 (1999). 
(a)

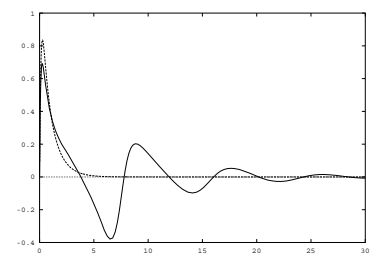

(b)

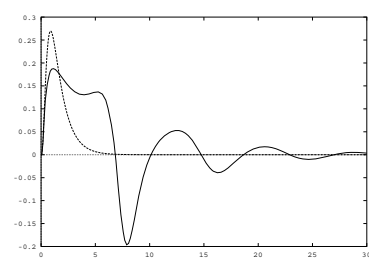

Figure 1: The numerical trajectory (solid line) $f_{2}(t)$ (panel a), and $f_{4}(t)$ (panel b), of the Smoluchowski ODE system (11), compared with the exact expectation (dashed line). The horizontal axis is time.

(a)

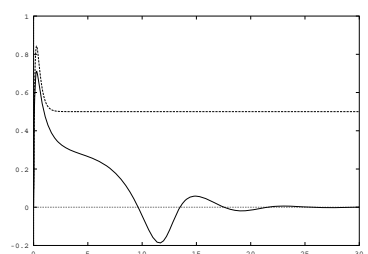

(b)

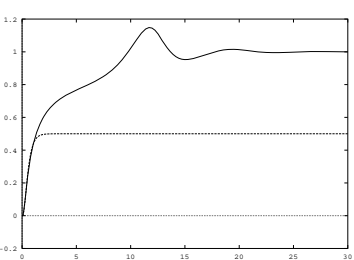

Figure 2: The numerical trajectory (solid line) $f_{2}(t)$ (panel a), and $f_{4}(t)$ (panel b), of the Smoluchowski ODE system (15), compared with the exact expectation (dashed line). The horizontal axis is time. 